Ortega Sánchez, D. \& Gómez Trigueros, I.M. (2017). Las Webquests y los MOOCs en la enseñanza de las Ciencias Sociales y la formación del profesorado de Educación Primaria. Revista Electrónica Interuniversitaria de Formación del Profesorado, 20(2), 205-220.

DOI: http://dx.doi.org/10.6018/reifop.20.1.258551

\title{
Las WebQuests y los MOOCs en la enseñanza de las Ciencias Sociales y la formación del profesorado de Educación Primaria.
}

\author{
Delfín Ortega Sánchez ${ }^{1}$, Isabel $M^{\mathrm{a}}$ Gómez Trigueros ${ }^{2}$ \\ ${ }^{(1)}$ Universidad de Burgos, ${ }^{(2)}$ Universidad de Alicante
}

\section{Resumen}

Este artículo analiza las experiencias y concepciones metodológicas del profesorado en formación de Educación Primaria sobre el aprendizaje colaborativo con WebQuests y MOOCs en el área curricular de Ciencias Sociales. El estudio centra su interés en la vinculación de estas dos herramientas didácticas a la adquisición de competencias básicas y su potencial para la capacitación en Conocimiento Didáctico del Contenido. Los resultados obtenidos demuestran la necesidad educativa de transferir e integrar, de forma operativa y funcional, el avance y generalización de las Tecnologías de la Información y de la Comunicación en los planes de estudio de formación del profesorado.

\section{Palabras clave}

MOOC; WebQuest; formación del profesorado; Ciencias Sociales.

\section{WebQuests and MOOCs in the teaching of the Social Studies and the Primary Education Teacher Training.}

\section{Abstract}

This article analyzes the experiences and methodological conceptions of the Primary Education Teacher Training on the collaborative learning with WebQuests and MOOCs in

Contacto:

Delfín Ortega Sánchez, dosanchez@ubu.es, Facultad de Educación, Universidad de Burgos, C/ Villadiego, s/n. 09001, Burgos. Isabel M Mámez Trigueros, isabel.gomez@ua.es, Facultad de Educación, Universidad de Alicante, C/ Aeroplano, s/n. 03690, San Vicente del Raspeig (Alicante).

Este artículo ha sido realizado al amparo del Grupo de Innovación Docente de la Universidad de Burgos en Didáctica de las Ciencias Sociales, de las Lenguas y de las Literaturas en la formación inicial del profesorado en Educación Infantil y Educación Primaria. 
the area curricular of Social Sciences. The study focus on these didactic tools to the acquisition of basic competences and to its potential for the training on Pedagogical Content Knowledge. The obtained results demonstrate the educational need to transfer and integrate, of operative and functional form, the advance and generalization of the Technologies of the Information and of the Communication in the plans of study of Teacher Training.

\section{Key words}

MOOC; WebQuest; Teacher Training; Social Studies.

\section{Introducción}

La Sociedad de la Información, del Aprendizaje o de la Inteligencia ofrece un volumen ingente de información de acceso inmediato, posibilita la comunicación instantánea entre sus usuarios (consumidores y productores, al tiempo, de esa misma información) y relativiza la procedencia geográfica y tiempos de producción de contenidos. La limitación de acceso al conocimiento y los principios científico-racionalistas del paradigma educativo tradicional (old smart) comienzan a apartarse para priorizar una ecología del conocimiento dinámica, regida por principios y expectativas más colaborativas, interactivas y de auto-creación del conocimiento guiado, que posicionan al sujeto en un rol activo en contextos comunitarios de inteligencia (Gómez y Ortega, 2014; Vázquez, López y Sarasola, 2013).

Internet ha influido, decisivamente, en la forma de aprender, trabajar y socializarse, al modificar el concepto de distancia y tiempo en el acceso a la información. Esta realidad ha requerido del desarrollo de nuevas competencias personales y sociales para la criba, discriminación crítica y posterior transformación de esta información en conocimiento, pues, en efecto, "transformar la información en conocimiento exige unas destrezas de razonamiento para organizar la información, relacionarla, analizarla, sintetizarla y hacer inferencias y deducciones de distinto nivel de complejidad" (Miralles, Gómez y Arias, 2013, p. 101).

La educación representa el marco base en el que articular las acciones necesarias para la puesta en marcha de la denominada alfabetización digital, impulsora, asimismo, del refuerzo de un paradigma del proceso educativo cada vez más orientado hacia el estudiante como agente activo de su propio proceso de aprendizaje. Esta alfabetización, igualmente, favorece la motivación, a partir de los recursos textuales, visuales, sonoros y comunicativos disponibles, desencadenantes de intereses e implicaciones efectivas del alumnado en los distintos programas educativos desarrollados en tiempos y ritmos más flexibles y autónomos. Para ello, resulta imprescindible la adquisición docente de las competencias didácticas, tecnológicas y tutoriales necesarias (Del Moral y Villalustre, 2012), con el objeto de alcanzar una integración tecnológica eficaz en el aula y para el desarrollo de habilidades tecnológico-digitales en el alumnado.

Desde esta perspectiva, la incorporación operativa de las Tecnologías de la Información y de la Comunicación (TIC) en el aula precisa de la adaptación a contextos educativos en continuo cambio, del fomento del trabajo cooperativo-colaborativo, del trabajo autónomo y del aprendizaje competencial para la resolución de problemas. Las TIC y el desarrollo de la competencia digital permiten, en entornos educativos, la interactividad en el proceso de enseñanza y aprendizaje, e impulsa la innovación docente. En este sentido, el trabajo por competencias prioriza el enfoque constructivista, fomenta el aprendizaje autónomo, el trabajo cooperativo-colaborativo, la transferencia de aprendizajes, la integración operativa de las TIC y la interdisciplinariedad, orientaciones a las que el profesorado deberá dar 
respuesta desde el diseño de los planes y programas de estudio de formación inicial (Guitert i Catasús, Romeu y Pérez-Mateo, 2007; Herts y Sempere, 2011; Palomares y Garrote, 2010).

En esta línea, las Webquests y los Cursos Online Masivos y Abiertos -en adelante, MOOCs-, unidos al crecimiento y generalización de las TIC, dan cuenta, de forma dirigida y satisfactoria, de las nuevas formas de enseñar y aprender o de pedagogías emergentes, validando su potencial didáctico, en consecuencia, en la formación inicial del profesorado de Educación Primaria.

\section{MOOC's y WebQuests en la enseñanza de las Ciencias Sociales: aproximación a su definición y dimensión educativa en la formación del profesorado.}

En los últimos años, un buen número de trabajos sobre la enseñanza de las Ciencias Sociales han venido insistiendo en la necesidad de integrar las TIC en la programación didáctica de aula, ofreciendo eficaces respuestas, mediante la utilización de técnicas de realidad virtual o la multimedia expositiva (Rivero, 2011), a las dificultades planteadas por las disciplinas humanísticas, como la imposibilidad de reproducir hechos históricos y sociales (Acosta, 2010), y en menor medida, fenómenos geográficos.

La integración de las TIC y el desarrollo de la competencia digital en la comprensión de fenómenos sociales y culturales, y en la adquisición de competencias sociales y ciudadanas, pueden fácilmente implementarse en el diseño de actividades, tareas o situaciones que permitan la búsqueda, obtención, selección, procesamiento, presentación y comunicación de información relativa a la cultura, la historia y el arte. Las simulaciones virtuales y las propuestas didácticas, programadas para el fomento de actitudes críticas y reflexivas sobre la información obtenida, resultan buenas estrategias para la integración curricular de las TIC en la enseñanza-aprendizaje de la Historia y de las Ciencias Sociales, y en el desarrollo de competencias básicas de eminente naturaleza social y cultural (Ortega, 2014). Sigue haciéndose necesaria, por tanto, la integración operativa de recursos, estrategias y metodologías TIC, capaces de construir conocimiento desde sus propias cualidades y aprovechamiento didáctico.

De acuerdo a las directrices del Ministerio de Educación, los planes de estudio de los Grados en Maestro/a en Educación Infantil y Primaria prescriben, explícitamente, la capacitación profesional de los nuevos profesionales en el uso de las TIC, así como su valoración social e integración curricular, competencia esta última considerada básica. Sin embargo, "no parece que al elaborar los planes de formación inicial del profesorado se haya partido ni de la ley de educación ni de las posibles necesidades de la sociedad futura" (Gutiérrez, 2012, p. 65). En efecto, la formación del profesorado debe seguir orientando sus esfuerzos hacia la adquisición de metodologías generadas a partir de los recursos y materiales didácticos que proporcionan las TIC, y de habilidades para la gestión y tratamiento de la información, más allá de la utilización como usuario de estas tecnologías (Del Moral y Villalustre, 2012; Ortega, 2014; Ortega, 2015). Buena prueba de ello son las dificultades que pueden comprobarse en el alumnado universitario en relación a la habilidad para incorporar herramientas TIC en su propio proceso formativo, aun cuando conviven con el uso habitual de este tipo de tecnologías (Barnes, Marateo y Ferris, 2007).

La presencia de las TIC en los planes de estudio de formación de maestros/as, impulsada por la aparición de las nuevas tecnologías y de sus pedagogías emergentes (Adell y Castañeda, 2012), habría de garantizar la adquisición y desarrollo de competencias tecnológicas (Monereo, 2005; Ortega, 2015), entendidas como "la capacidad, conocimiento 
y actitud de uso de tecnologías de la información y la comunicación en sus diversas funciones y contextos de aplicación" (Cózar, 2013, p. 46). La capacitación docente en TIC y sus procesos formativos, ha de incluir además, desde perspectivas sinérgicas e interactivas, las competencias propiamente disciplinares y pedagógicas, alcanzando lo que ha venido en denominarse el Conocimiento Técnico Pedagógico del Contenido o modelo TPACK (Technological Pedagogical Content Knowledge) (Cózar, Sagalaz y Sáez, 2015a; Del Moral y Villalustre, 2010; Gómez, 2015; Koehler y Mishra, 2008). En efecto, la asunción de los nuevos roles del alumnado y del profesorado, que la educación hoy demanda en los nuevos planteamientos de los procesos de enseñanza-aprendizaje (Trigueros, Molina, Sánchez, Valverde y Agulló, 2010), junto a la tradicional capacitación disciplinar, exige del docente la capacitación instrumental y didáctico-tecnológica necesaria con la que aproximarse, de forma crítica y reflexiva, a los recursos y herramientas digitales.

Recientemente, Cózar et al. (2015a) llamaban la atención sobre la eliminación de asignaturas troncales como las Nuevas tecnologías aplicadas a la Educación de los planes de estudio universitarios de maestro/a, "relegando la formación en TIC a optativas o a un tratamiento transversal a criterio de los docentes de unas pocas asignaturas" (p. 150). Esta ausencia curricular deriva en alarmantes resultados de investigación, vertidos en recientes estudios dedicados al análisis de las representaciones, conocimientos, percepciones y usos sobre TIC del profesorado de Ciencias Sociales en formación (Trigueros, Sánchez y Vera, 2012), y en la necesidad de implementar programaciones específicas de aula para la adquisición de la competencia digital docente de maestros/as en formación inicial. Con el objeto de mejorar el proceso de enseñanza y aprendizaje de las disciplinas sociales en Educación Primaria, estos trabajos insisten en la utilización programada e intencionada de las TIC (Sangrà, 2011), integrada en nuevos escenarios de aprendizaje para la adquisición de habilidades y competencias docentes y digitales. En esta línea, son destacables las aportaciones de investigaciones sobre experiencias didácticas realizadas en formación del profesorado desde la Didáctica de las Ciencias Sociales (Cózar, 2013; Cózar, Hernández, Hernández, De Moya y Guerrero, 2014; Cózar et al., 2015a; Cózar, De Moya, Hernández y Hernández, 2015b; Gómez, 2015; Gómez y Ortega, 2014; Miralles et al., 2013; Moreno, Vera y López, 2014; Ortega, 2014, 2015; Ortega y Gómez, 2015; Ortega, Gómez y Moreno, 2016; Roblizo y Cózar, 2015).

\subsection{WebQuests, estrategias de trabajo cooperativo-colaborativo en la formación del profesorado.}

Como evidencian los resultados de investigación sobre las TIC en la formación inicial del profesorado de Ciencias Sociales (Cózar, 2013; Miralles et al., 2013; Ortega, 2014), las Webquests constituyen una excelente herramienta para la adquisición de habilidades de gestión y tratamiento de la información, así como de competencias propiamente disciplinares, pedagógicas y tecnológicas, en torno a la resolución de problemas mediante metodologías de indagación/investigación y de aprendizaje basado en tareas.

Los creadores de esta estrategia, Dodge (1995) y March (2003), la describen como "una actividad de investigación en la que la información con la que interactúan los alumnos proviene total o parcialmente de recursos de Internet" (Dodge, 1995). Podría afirmarse que se trata de una "unidad de aprendizaje pautada, que consiste en el desarrollo de un proyecto de trabajo de distinta naturaleza (investigación, análisis, etc.) realizado a partir de la consulta de webs previamente seleccionadas por el docente" (Osset, 2013), o una "estrategia de aprendizaje por descubrimiento guiado a un proceso de trabajo desarrollado por los alumnos utilizando los recursos de la www", que integra "los principios del aprendizaje constructivista, la metodología de enseñanza por proyectos y la navegación 
web para desarrollar el currículum con un grupo de alumnos en una aula ordinaria" (Area, 2004).

La WebQuest propone una tarea factible, significativa y atractiva para los estudiantes desde un planteamiento problematizado (productos de aprendizaje) y un proceso, basado en actividades dirigidas al tratamiento de la información (análisis, síntesis, creación, evaluación, etc.), para realizarla (Adell, 2004; Barba, Bernabé, Adell y Capella, 2008; Pérez, 2004). Su diseño y aplicación deberá ser capaz de fomentar el desarrollo de habilidades cognitivas de orden superior, transformando la información en conocimiento de forma cooperativa-colaborativa (Lara y Repáraz, 2007). De acuerdo a sus creadores, una posible taxonomía de tareas contempla actividades de autoconocimiento, científicas, de juicio, diseño, repetición, de misterio, analíticas, de compilación o de consenso, entre otras.

La selección de recursos web por el docente revierte en la supresión del tiempo invertido en su localización por parte del alumnado para priorizar el uso, tratamiento, procesamiento y posterior transformación de la información, tras el seguimiento de un proceso de indagación/investigación dirigido al fomento del pensamiento crítico, la toma decisiones y la creatividad.

La metodología que define la estrategia de las WebQuests se basa en el aprendizaje por descubrimiento, parte del método científico, prioriza el propio proceso de aprendizaje (no un contenido concreto) e impulsa la participación activa y autónoma del alumnado que, organizado en grupos y con una distribución de roles definidos, se presenta como el verdadero artífice de su propio conocimiento, bajo la orientación/guía del maestro/a.

El diseño estructural de la estrategia, secuenciado en Introducción, Tarea, Proceso, Recursos, Evaluación y Conclusión, supone "una de las principales características que permite la investigación guiada, facilitando la organización y seguimiento de la tarea" (Cózar, 2013, p. 48), ofreciendo múltiples ventajas para una integración funcional de las TIC en el aula de Primaria, el desarrollo de la competencia para aprender a aprender, el trabajo cooperativocolaborativo y la creatividad del alumnado (Temprano, 2009).

En definitiva, el desarrollo del trabajo intelectual, impulsado en esta estrategia, atiende, de forma prioritaria, al tratamiento y gestión de la información y no a su búsqueda, previamente programada por el docente, concentrando el interés en su análisis, síntesis y evaluación. Igualmente, utiliza técnicas de simulación o adjudicación de roles que favorecen la introducción del alumnado a la metodología de investigación de los estudios sociales e históricos, proponiendo al estudiante como protagonista de una situación problematizada del presente o del pasado y cuya resolución, materializada en la elaboración de un producto final (la tarea), dependerá de los resultados del trabajo colaborativo grupal (Vivancos, 2008). Tanto el proceso como el producto final serán publicados en la red, estímulo motivador para el equipo de trabajo.

\subsection{Los MOOCs y la formación del profesorado.}

Los MOOCs son considerados, como otros entornos virtuales, parte del software educativo 3.0. Esta herramienta, basada en los principios conectistas de masividad, gratuidad, portabilidad, ubicuidad, autoevaluación, modularidad y vídeo-simulación, propone nuevos escenarios de enseñanza-aprendizaje, más allá del tradicional espacio aula-práctica docente. Esta nueva forma de entender la construcción del conocimiento se orienta, como también sucede en las estrategias WebQuests, hacia la producción de contenidos y se fundamenta en el principio de enseñanza interactiva-colaborativa.

Los MOOCs, concebidos como entornos conectistas, colaborativos, guiados y flexibles de aprendizaje, reúnen dos principios básicos: el acceso abierto y gratuito, en el que los 
estudiantes no necesitan de la formalización de una matrícula en un Centro o institución educativa; y la interactividad y naturaleza colaborativa de los cursos, condicionadas por las dudas y planteamientos de sus usuarios (escalabilidad) (Gómez y Ortega, 2014; Ortega y Gómez, 2015).

A pesar de la abrumadora difusión de cursos MOOCs durante 2013 (Poy y Gonzáles-Aguilar, 2014), a través de plataformas como Unx, MiríadaX, UPVX y UnedComa -las de mayor relevancia en España-, sorprende la inexistencia de cursos desarrollados desde el ámbito de la Didáctica de las Ciencias Sociales y la formación del profesorado; vacío parcialmente resuelto con el diseño, realización e implementación del primer curso MOOC en España Curso o. Didáctica de la Geografía a través de Google Earth (http://didacticageografiaytic.uaedf.ua.es/preview). Para el diseño y configuración estructural de participación de este MOOC fueron considerados los modelos de Conole (2013), Montero y Viñuales (2013) y Raposo (2014), junto a los cuatro principios de Downes (2013): autonomía, por la que cada usuario (maestros/as en formación) ha de establecer sus propios objetivos y niveles de éxito en su participación en el MOOC; diversidad y apertura, definiendo la propuesta hacia su internacionalización, y adaptación espacio-temporal y cultural; e interactividad, base del aprendizaje en $M O O C$, en función de la propia conectividad en la relación participante/plataforma-recursos, y entre participantes (Gómez y Ortega, 2014).

No obstante, esta importante acogida de los MOOCs no la exime de sus debilidades. Junto a las dificultades de identificación del usuario inscrito y de certificación del seguimiento del curso, destaca la ausencia de sistemas de evaluación externos que permitan valorar la calidad educativa de los MOOCs, particularmente, de contenido social, geográfico e histórico (Ortega et al., 2016). En este sentido, ya han sido propuestos mecanismos de coevaluación, basados en los sistemas Peer to Peer ( $\mathrm{P} 2 \mathrm{P})$ y Karma, este último dirigido a la adjudicación cooperativa de puntos positivos por los usuarios del curso.

La proximidad conceptual de los MOOCs a la estrategia WebQuest, y su aprovechamiento conjunto en los programas de formación del profesorado, radica en sus potencialidades didácticas y pedagógicas, a partir de la creación/producción de contenidos, y de la actuación activa, interactiva y colaborativa del alumnado. Mediante estas dos herramientas, especialmente eficaces en la adquisición y desarrollo de competencias docentes digitales, el estudiante de maestro/a trasciende, por tanto, los roles pasivo-receptores para proponerse como agente activo y constructor de su propio proceso de aprendizaje desde las tres dimensiones competenciales apuntadas, también para el caso de las WebQuests: competencia técnica (TK), habilidades de facilitación (PK) y conocimiento disciplinar (CK) (Gómez, 2015).

Se puede afirmar que desde las diferentes instituciones (EEES, MEC, Gobiernos Autonómicos, etc.) se incide en la necesidad de formar en competencias clave, haciendo imprescindible su incorporación en la formación inicial del profesorado $y$, de forma particular, en el ámbito de las tecnologías. La actual Sociedad de la Información y la Comunicación (SIC) demanda profesionales capaces de utilizar e implementar las TIC en sus prácticas docentes diarias. Por todo ello, se convierte en tarea imprescindible conocer y analizar el nivel de competencia digital de los maestros/as en formación para, de este modo, adecuar y repensar los planes docentes universitarios.

El objetivo de esta investigación se centra en analizar el grado de conocimiento de los futuros docentes en tecnologías tales como los MOOC's y las WebQuest. Asimismo, se pretende valorar la actitud y conocer la percepción de la muestra participante respecto de 
estos recursos tecnológicos como herramientas de enseñanza y aprendizaje, evaluando su predisposición a la implementación de tales recursos TIC en el aula de Ciencias Sociales.

\section{Material y método.}

\section{1. Participantes.}

La muestra, no probabilística, no aleatoria e intencional por convivencia, se compuso de 105 estudiantes, número representativo según las indicaciones sobre el mínimo deseable para este tipo de estudios en Ciencias Sociales con cuestionarios (Sevillano, 2007). En relación al género, existe un predominio de mujeres como se observa en la Tabla 1, matriculadas en materias propias de la Didáctica de las Ciencias Sociales del Grado de Maestro/a en Educación Primaria de la Universidad de Burgos y de la Universidad de Alicante durante el curso 2014/2015.

Tabla 1.

Distribución de la muestra por género.

\begin{tabular}{|c|c|c|}
\hline Género & Número & $\%$ \\
\hline Mujer & 80 & 76,2 \\
\hline Hombre & 25 & 23,8 \\
\hline Total & 105 & 100,0 \\
\hline
\end{tabular}

En referencia a los cursos que realizan, se debe señalar que un $82,9 \%$ se encontraba matriculado en el tercer curso del Grado (Tabla 2).

Tabla 2.

Curso que realiza la muestra encuetada.

\begin{tabular}{|c|c|c|}
\hline \multicolumn{3}{|c|}{ Curso } \\
\hline & Frecuencia & $\%$ \\
\hline $2^{\circ}$ curso de Grado de Primaria & 9 & 8,6 \\
\hline $3^{\circ}$ curso de Grado de Primaria & 87 & 82,9 \\
\hline $4^{\circ}$ curso de Grado de Primaria & 4 & 3,8 \\
\hline Otros & 5 & 4,8 \\
\hline Total & 105 & 100,0 \\
\hline
\end{tabular}

Respecto al rango de edad, la muestra se distribuyó entre los 18 y 27 años, destacando el intervalo de los 18 a los 21 años (Tabla 3).

Tabla 3.

Distribución de la muestra por rango de edad.

\begin{tabular}{|c|c|c|}
\hline Rango de edad & Frecuencia & $\%$ \\
\hline de $18-21$ & 66 & 62,9 \\
\hline de $22-23$ & 14 & 13,3 \\
\hline
\end{tabular}




\begin{tabular}{|c|c|c|}
\hline de 24 o más & 25 & 23,8 \\
\hline Total & 105 & 100,0 \\
\hline
\end{tabular}

\section{2. Instrumento.}

Los datos objeto de análisis fueron obtenidos mediante la aplicación de un cuestionario, elaborado a partir de 11 ítems, 3 de ellos identificativos (género, rango de edad, curso de Grado que realiza) y 8 categoriales. Estos últimos se clasificaron en dos bloques: un primer bloque de cuatro cuestiones dirigidas al conocimiento conceptual, experiencial y metodológico de los MOOCs, y un segundo bloque, de tres cuestiones, dirigido a las WebQuests. Ambos se completaron con una cuestión conjunta que aunó la percepción de los maestros/as en formación de la viabilidad de las dos herramientas como recurso didáctico. Las respuestas posibles adoptaron la escala Likert, concretada en las siguientes estimaciones: En desacuerdo, Ni de acuerdo ni en desacuerdo, De acuerdo, Totalmente de acuerdo. Las respuestas emitidas fueron devueltas de forma anónima, a través de la plataforma web Formularios Google.

Como referencia para la elaboración del cuestionario, se han tomado las indicaciones de Best (1974) vigentes en la actualidad en los ámbitos de educación, así como los propuestos por Lozano, García-Cueto y Muñiz, (2008). El instrumento ha sido adaptado al objetivo concreto de esta investigación y se ha validado por expertos universitarios de los departamentos de Geografía Humana y Didáctica General y Específica de las dos universidades participantes en la investigación.

Para la codificación y procesamiento de datos, se aplicó el software estadístico SPSS (versión 23). La consistencia interna y fiabilidad del instrumento fue medida mediante el coeficiente Alpha de Cronbach, resultando el valor positivo $a=0,834$ (Sevillano, 2007), indicativo de una alta fiabilidad, credibilidad y fuerza a los resultados obtenidos y presentados en este trabajo. Asimismo, se ha procedido a la realización de análisis de ChiCuadrado de Pearson con resultados de p-valor<1= Sig. 0,001, (Cohen y Manion, 2002), mostrando una elevada correlación entre las preguntas propuestas. Todo ello permite afirmar la validez de las cuestiones y la estructura de la encuesta realizada.

\section{3. Diseño y procedimiento.}

La investigación se adscribe a enfoques metodológicos descriptivos-cuantitativos no experimentales en el que se recogen datos relativos al nivel de conocimiento y competencia tecnológica en MOOC's y Webquest, así como la actitud frente a estas herramientas por parte de la muestra participante. Para su desarrollo se han incorporado elementos propios del enfoque cuantitativo, necesario para poder analizar los resultados del cuestionario aplicado (Hernández, Fernández y Baptista, 2003).

El estudio ha sido realizado en el contexto de las Facultades de Educación de las Universidades de Burgos y de Alicante durante el curso académico 2014-2015, en el ámbito de las asignaturas de Didáctica de las Ciencias Sociales del Grado de Maestro/a de Educación Primaria.

Al tratarse de un estudio exploratorio y no experimental, el procedimiento seguido partió de la distribución de un cuestionario on-line al alumnado matriculado, a través de la plataforma gratuita Formulario de Google, con la intención de recabar datos sobre la formación en competencia TIC de los futuros docentes y conocer su percepción sobre la capacidad didáctica de las herramientas tecnológicas mencionadas. Durante el proceso de aplicación del cuestionario, se garantizó el anonimato de la muestra participante y la confidencialidad en el tratamiento de datos. Posteriormente, se ha procedido al vaciado y 
análisis estadístico descriptivo de las respuestas recibidas, atendiendo a las frecuencias, desviación típica (Dt.) y media ( $\overline{\mathrm{X}}$ ) de las variables contempladas en este estudio.

\section{Resultados.}

En relación al conocimiento sobre el concepto MOOC y WebQuest que poseen los futuros docentes, se constatan respuestas poco positivas, indicativas del desconocimiento respecto a estas herramientas tecnológicas, en particular, hacia los MOOCs. Así, al ítem 4: "Conozco el concepto MOOC y comprendo su significado", el 60\% de los/as encuestados/as responde En desacuerdo y sólo el 24,8\% se muestran De acuerdo. Asimismo, al ítem 5: "He participado en un MOOC como estudiante", el 71,4\% responden En desacuerdo. Con respecto a los lugares desde los que acceder a estos cursos on-line, las respuestas son significativas, pues más del $60 \%$ de los/as participantes manifiesta su desconocimiento sobre las plataformas donde se aloja la oferta formativa de MOOCs (ítem 6).

Si se comparan dichos resultados con las respuestas obtenidas en el ítem 8 , referente al concepto WebQuest, se aprecia que sólo el 32,4\% se decanta por la opción En desacuerdo, mientras que el 55,2\% dan por respuesta De acuerdo. En relación a su utilización, los resultados del ítem 9: "He utilizado WebQuest (como diseñador y/o como participante)", indican que su uso es más generalizado y conocido que los MOOCs, con el registro de un $44,8 \%$ de respuestas De acuerdo.

Los factores causales de tales resultados (Figura 1) pueden explicarse en la familiarización del alumnado con el uso de la WebQuest, ya que esta TIC se utiliza como herramienta de enseñanza y aprendizaje en la formación inicial del profesorado de Ciencias Sociales en las Facultades de Educación de las Universidades de Burgos y Alicante. Estos resultados, en cambio, no son parejos a los MOOCs, menos conocidos y con un menor desarrollo entre los estudiantes del Grado de Primaria.

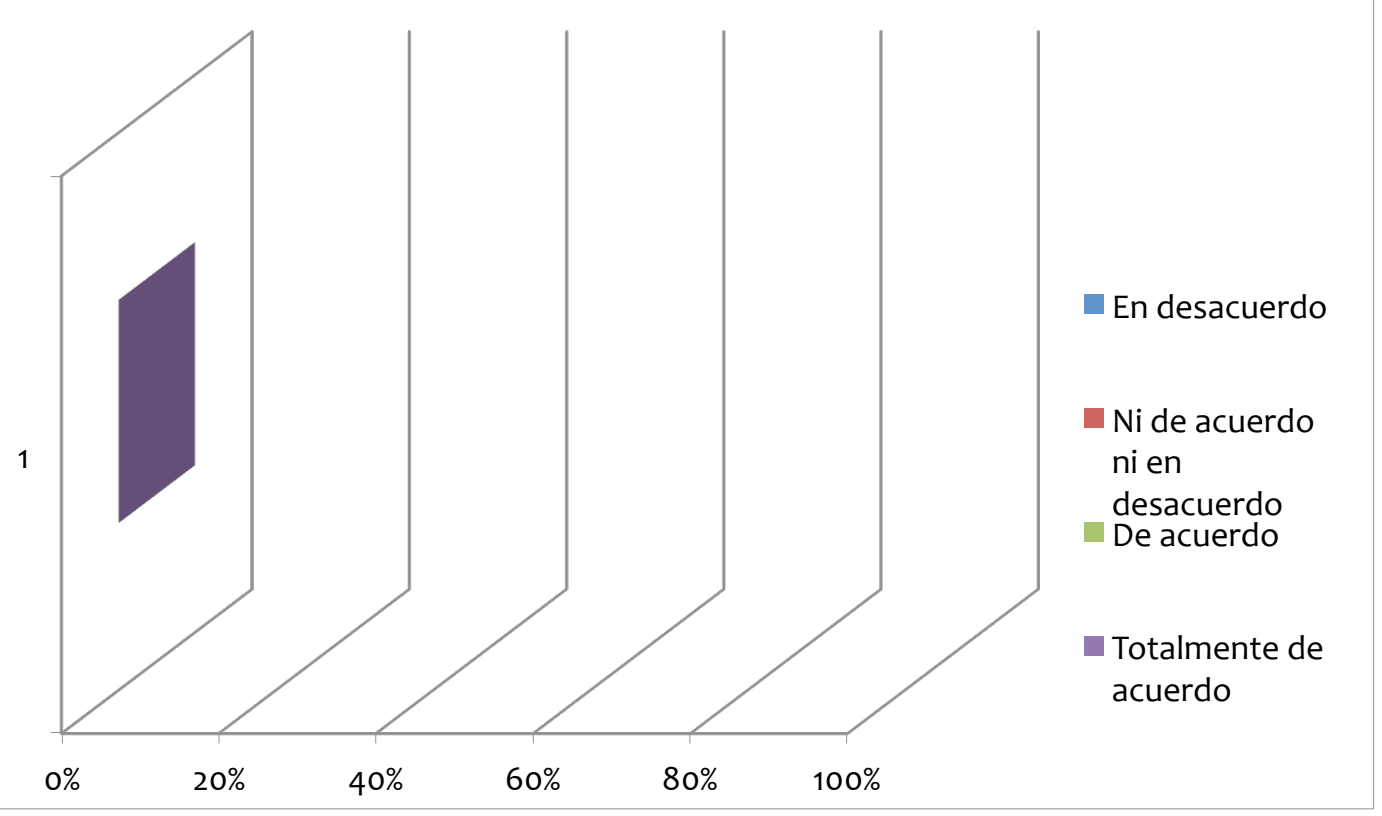

Figura 1. Análisis sobre el conocimiento del MOOC y WebQuest. 
En cuanto a la percepción del alumnado participante sobre el potencial didáctico de las dos herramientas, resultan especialmente representativas las respuestas generadas a partir del ítem 11: "Los MOOCs y las WebQuest son recursos útiles para la enseñanza y el aprendizaje de las Ciencias Sociales", de donde se desprende que el 42,9\% de los/as encuestados/as está De acuerdo con tal afirmación, frente a al 4,8\% que se muestran En desacuerdo (Figura 2).

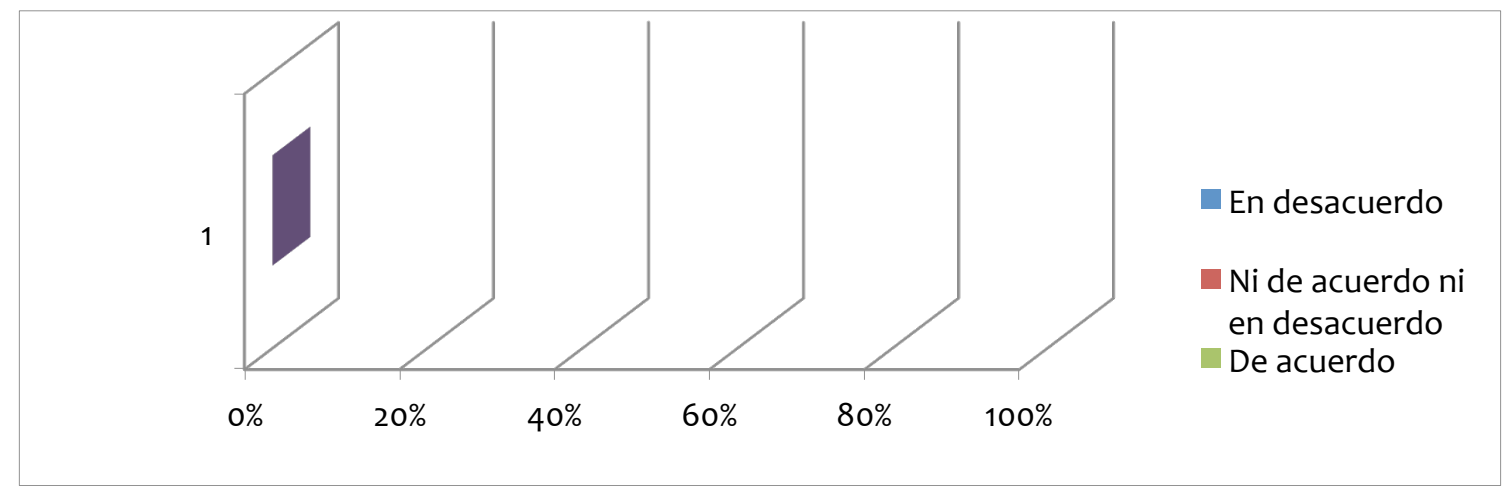

Figura 2. Valoración formativa y didáctica del MOOC y WebQuest.

En referencia a la desviación típica (Tabla 4), consideramos que los resultados arrojados por el cuestionario son adecuados, pues todos ellos se encuentran por debajo del valor 1 (Dt.<1), cumpliéndose, de este modo, el criterio propuesto por Rowntree (1981), quien sugiere que, en muestras de tamaño superior a 100, la Dt. no ha de ser más elevada que una quinta parte de la escala. Si observamos los valores de media, se aprecia una dicotomía entre las preguntas referidas al MOOC y las relacionadas con las WebQuest.

Tabla 4.

Conocimiento y percepción sobre las herramientas TIC WebQuest y MOOCs.

\begin{tabular}{|l|c|c|c|}
\hline \multicolumn{3}{|c|}{ Estadísticas de elemento } \\
\hline & $\begin{array}{c}\text { Media } \\
(\bar{x})\end{array}$ & $\begin{array}{c}\text { Desviación } \\
\text { estándar } \\
(\mathrm{Dt} .)\end{array}$ & $\mathrm{N}$ \\
\hline P4. Conozco el concepto MOOC. & 1,65 & 0,755 & 105 \\
\hline P5. He participado en un MOOC como estudiante. & 1,40 & 0,588 & 105 \\
\hline $\begin{array}{l}\text { P6. Conozco algunas plataformas en las que se alojan y } \\
\text { presentan MOOCs. }\end{array}$ & 1,57 & 0,707 & 105 \\
\hline $\begin{array}{l}\text { P7. Considero que los MOOCs me ayudan en mi } \\
\text { formación como futuro maestro/a. }\end{array}$ & 2,04 & 0,678 & 105 \\
\hline $\begin{array}{l}\text { P8. Conozco el concepto de WebQuest y comprendo su } \\
\text { significado. }\end{array}$ & 2,23 & 0,912 & 105 \\
\hline $\begin{array}{l}\text { P9. He utilizado WebQuest (como diseñador/a y/o } \\
\text { participante). }\end{array}$ & 2,24 & 0,839 & 105 \\
\hline $\begin{array}{l}\text { P10. Considero que las WebQuest me ayudan en mi } \\
\text { formación como futuro docente. }\end{array}$ & 3,36 & 0,537 & 105 \\
\hline $\begin{array}{l}\text { P11. Los MOOCs y las WebQuest son recursos útiles para } \\
\text { la enseñanza y el aprendizaje de la Ciencias Sociales. }\end{array}$ & 2,88 & 0,578 & 105 \\
\hline
\end{tabular}


Así, en los ítems 4, 5 y 6 observamos resultados por debajo de $2(\bar{X}<2)$, excepto en la cuestión 7, donde la media es mayor a 2. Tales valores confirman el desconocimiento que los estudiantes de $2^{\circ}, 3^{\circ}$ y $4^{\circ}$ curso manifiestan sobre estas TIC, a pesar del reconocimiento de su potencial formativo.

$\mathrm{Si}$, por el contrario, analizamos los valores de media de los ítems 8, 9 y 10, relacionados con la WebQuest, los valores son todos superiores a $2(\bar{X}>2)$. El dato más elevado se halla en el ítem 10 ( $\bar{X}>3)$. Dicho valor hace referencia a la categoría De acuerdo y se puede explicar desde el elevado papel formativo que para los discentes tiene esta herramienta tecnológica. Otra posible explicación a tales resultados se relaciona con la mayor implementación de las WebQuest en la formación inicial del profesorado como recursos TIC para la enseñanza y el aprendizaje en los Centros universitarios participantes.

Respecto al ítem 11, éste arroja una media superior a $2(\bar{X}>2)$, próxima al valor 3 , que se corresponde con la categoría De acuerdo. Resulta significativa la percepción positiva de los/as participantes en relación al rol formativo de las TIC propuestas. Estos resultados inciden en la consideración de los MOOCs y de las WebQuest como recursos activos, tanto para la enseñanza y formación del futuro profesorado, como para su posterior utilización como herramienta de aprendizaje en el área de Ciencias Sociales.

\section{Discusión y conclusiones.}

Los resultados analizados presentan valores bajos o muy bajos en relación al conocimiento de los recursos tecnológicos objeto de estudio (MOOC y WebQuest) y al acceso a las plataformas en Internet que los alojan. Los indicadores demuestran la escasa implementación de estas TIC en el aula universitaria para la formación de maestros/as. En contraposición, se observan altos valores en cuanto a la percepción formativa de estas herramientas, de su utilidad en los procesos de enseñanza y aprendizaje, y en la adquisición y tratamiento didáctico de contenidos propios de las Ciencias Sociales. Estos resultados coinciden con las aportaciones de investigaciones similares, en donde se analiza el valor educativo de las WebQuest (Ramos, 2009; Zubillaga y Alba, 2005) y del MOOC (Gómez y Ortega, 2014).

En la actualidad, ya son numerosos los trabajos que analizan y evalúan la dimensión educativa de herramientas y recursos tecnológicos como el MOOC y la WebQuest. En este sentido, son patentes sus fortalezas, y también sus debilidades, a la hora de transmitir contenidos y desarrollar competencias, no solo en el uso de las TIC (Ortega y Gómez, 2015; Revuelta y Esnaola, 2013), sino también en la selección de información, el trabajo colaborativo (Lara y Repáraz, 2007) y el aprendizaje entre iguales (Allan y Street, 2007; Gómez y Ortega, 2014).

Paralelamente, se vienen desarrollando investigaciones en las que se pone de manifiesto la escasa o nula formación de los futuros maestros/as en el manejo de las herramientas tecnológicas desde de una perspectiva docente (Cózar et al., 2015a; Prendes, Castañeda y Gutiérrez, 2010; Roblizo y Cózar, 2015). Tales conclusiones son coincidentes con los resultados obtenidos en el presente trabajo. El estudio, realizado sobre una muestra de 105 futuros profesores/as, evidencia la imperante necesidad de adecuar los actuales planes de estudio de Grado de Primaria a los nuevos requerimientos de la sociedad actual, pues, como señala Cózar et al. (2015a), se trata de futuros docentes que habrán de trabajar con recursos tecnológicos para desarrollar su labor profesional. 
Las normativas curriculares de las distintas etapas educativas concretan la necesidad de integrar las TIC en los procesos de enseñanza-aprendizaje de forma transversal a las áreas de conocimiento. Igualmente, el diseño de los distintos planes de estudio de los Grados en Maestro/a en Educación Infantil y Primaria garantiza la capacitación profesional de los nuevos maestros y maestras en el uso de las TIC. Sin embargo, como demuestran diversos estudios especializados, no parece que los planes de formación inicial del profesorado atiendan, en esta dirección, a las líneas educativas oficiales ni, por tanto, a las posibles necesidades de la sociedad futura.

En definitiva, podemos afirmar que la adquisición y desarrollo de competencias docentes digitales en la formación de maestros y maestras de Educación Primaria, coherente con los aprendizajes cooperativos on-line entre iguales, necesariamente pasa por la aproximación formativa a herramientas TIC (como el MOOC y la WebQuest), capaces de proponer distintas formas de acceso al conocimiento y a la autoformación, y de adaptarse a las actuales exigencias formativas en competencias de forma completa y conectada con la realidad del siglo XXI.

\section{Referencias bibliográficas}

Acosta, L. M. (2010). El tratamiento de la información y competencia digital (TICD) en la enseñanza-aprendizaje de la Historia en Bachillerato. CATHARUM Revista de Ciencias y Humanidades del Instituto de Estudios Hispánicos de Canarias, 11, 57-67.

Adell, J. (2004). Internet en el aula: Las WebQuest. Edutec. Revista Electrónica de Tecnología Educativa, 17. Recuperado de http://www.edutec.es/revista/index.php/edutece/article/view/530/264

Adell, J. y Castañeda, L. (2012). Tecnologías emergentes, ¿pedagogías emergentes? En J. Hernández, M. Pennesi, D. Sobrino y A. Vázquez (Eds.), Tendencias emergentes en educación con TIC (pp. 13-32). Barcelona: Asociación Espiral, Educación y Tecnología.

Allan, J. y Street, M. (2007). The Quest for Deeper Learning: An Investigation into the Impact of a Knowledge-Pooling WebQuest in Primary Initial Teacher Training Preview. British Journal of Educational Technology, 38(6), 1102-1112.

Area, M. (2004). Webquest. Una estrategia de aprendizaje por descubrimiento basada en el uso de Internet. Quaderns Digitals. Monográfico Webquests, 32. Recuperado de http://www.quadernsdigitals.net

Barba, C., Bernabé, I., Adell, J. y Capella, S. (2008). Les WebQuest en l'educació infantil i primària. Barcelona: UOC.

Barnes, K., Marateo, R. C. y Ferris, S. P. (2007). Teaching and learning with the net generation. Innovate: Journal of Online Education, 3(4), 1-8. Recuperado de http://nsuworks.nova.edu/innovate/vol3/iss4/1

Best, J. (1974). Como Investigar en Educación. Madrid: Aguilar.

Cohen, L. y Manion, L. (2002). Métodos de investigación educativa. Madrid: La Muralla.

Conole, G. (2013). Los MOOCs como tecnologías disruptivas: estrategias para mejorar la experiencia de aprendizaje y la calidad de los MOOCs. Campus Virtuales. Revista Científica de Tecnología Educativa, II(2), 16-28. 
Cózar, R. (2013). Las Webquest en la enseñanza/aprendizaje de las Ciencias Sociales. En R. Cózar y M. de V. de Moya (Coords.), Las TIC en el aula desde un enfoque multidisciplinar. Aplicaciones prácticas (pp. 45-62). Barcelona: Octaedro.

Cózar, R., De Moya, Ma del V., Hernández, J. A. y Hernández, J. R. (2015b). Tecnologías emergentes para la enseñanza de las Ciencias Sociales. Una experiencia con el uso de realidad aumentada en la formación inicial de maestros. Digital Education Review, 27, 138-153.

Cózar, R., Hernández, J. A., Hernández, J. R., De Moya, Ma del V. y Guerrero, O. (2014). La integración de las TIC en el aula desde la formación del profesorado de Educación Infantil y Primaria. En J. J. Maquilón y N. Orcajada (Eds.), Investigación e Innovación en formación del profesorado (pp. 423-432). Murcia: Universidad de Murcia.

Cózar, R., Zagalaz, J. y Sáez, J. M. (2015a). Creando contenidos curriculares digitales de Ciencias Sociales para Educación Primaria. Una experiencia TPACK para futuros docentes. Educatio Siglo XXI, 33(3), 147-168.

Del Moral, M. E. y Villalustre, L. (2010). Formación del Profesor 2.0: desarrollo de competencias tecnológicas para la escuela 2.0. Magister. Revista Miscelánea de Investigación, 23, 59-70.

Del Moral, $M^{a}$ E. y Villalustre, L. (2012). Didáctica universitaria en la era 2.0: competencias docentes en campus virtuales. RUSC. Revista Universidad y Sociedad del Conocimiento, 9(1), 36-50.

Dodge, B. J. (1995). Some thoughts about WebQuests. Recuperado de http://edWeb.sdsu.edu/courses/edtec596/about_Webquests.html

Downes, S. (2013). The Quality of Massive Open Online Courses by Stephen Downes. Recuperado de http://mooc.efquel.org/week-2-the-quality-of-massive-open-onlinecourses-by-stephendownes/

Gómez, I. Ma (2015). Proyecto a partir del modelo TPACK para desarrollar el aprendizaje de la Geografía en los estudios de Grado de Educación Primaria (Tesis Doctoral). Universidad de Alicante, Alicante.

Gómez, I. Mª y Ortega, D. (2014). Los MOOC en la Didáctica de la Geografía: Aplicaciones en la formación inicial del profesorado de Primaria. En R. Martínez y E. Tonda (Eds.), Nuevas perspectivas conceptuales y metodológicas para la educación geográfica (pp. 229-244). Córdoba: Universidad de Córdoba.

Guitert i Catasús, M., Romeu, T. y Pérez-Mateo, M. (2007). Competencias TIC y trabajo en equipo en entornos virtuales. RUSC. Universities and Knowledge Society Journal, 4(1), $1-12$.

Gutiérrez, A. (2012). Formación del profesorado para la alfabetización múltiple. En M. Area, A. Gutiérrez y F. Vidal (auts.), Alfabetización digital y competencias informacionales (pp. 43-98). Madrid-Barcelona: Ariel-Fundación Telefónica.

Hernández, R., Fernández, C. y Baptista, P. (2003). Metodología de la Investigación. México: Mc Graw-Hill.

Herts, A. y Sempere, M. A. (2011). Nuevos modelos de formación para el trabajo por competencias. En Conectando Redes (pp. 693-732). Madrid: Ministerio de Educación.

Koehler, J. y Mishra, P. (2008). What is technological pedagogical content knowledge (TPCK)? In AACTE Committee on Innovation and Technology (Eds.), Handbook of 
Technological Pedagogical Content Knowledge (TPCK) for Educators (pp. 1-30). New York: Routledge/Taylor y Francis Group.

Lara, S. y Repáraz, Ch. (2007). Eficacia de las WebQuest para el aprendizaje cooperativo. Revista Electrónica de Investigación Psicoeducativa, 5-3(13), 731-756.

Li, S., Luk, T. K. y Jong, S. Y. (2014). Design of MOOC for In-service Teacher Professional Development: A Case of Teachers' Refresher Training Course in Hong Kong. In T. Supnithi, S. C. Kong, Y. T. Wu, T. Kojiri, C. C. Liu, H. Ogata, y A. Kashihara (Eds.), Proceedings of the $22^{\text {nd }}$ International Conference on Computers in Education (pp. 5085013). Japan: Asia-Pacific Society for Computers in Education.

Lozano, L. M., García-Cueto, E. y Muñiz, J. (2008). Effect of the number of response categories on the reliability and validity of rating scales. Methodology, 4, 73-79.

March, T. (2003). The learning power of WebQuest. Educational Leadership, 61(4), 42-47.

Miralles, P., Gómez, C. J. y Arias, L. (2013). La enseñanza de las ciencias sociales y el tratamiento de la información. Una experiencia con el uso de webquests en la formación del profesorado de Educación Primaria. Revista de Universidad y Sociedad del Conocimiento (RUSC), 10(2), 98-111.

Monereo, C. (Coord.) (2005). Internet y competencias básicas. Aprender a colaborar, a comunicarse, a participar, a aprender. Barcelona: Graó.

Montero, I. y Viñuales, J. (2013). Difundi y OpenMOOC. Desarrollando un modelo de negocio basado en servicios MOOC y sobre una plataforma de software libre. En Scopeo. Informe $n^{\circ}$ 2. MOOC: estado de la situación actual, posibilidades, retos y futuro (pp. 140-155). Salamanca: Universidad de Salamanca.

Moreno, J. R., Vera, M ${ }^{\mathrm{a}}$ I. y López, I. (2014). Develpment of creative and educational thinking in arts training teachers: QR codes. Sylwan Journal, 158(12), 185-200.

Ortega, D. (2014). Las TIC y el desarrollo de competencias básicas en la enseñanza del Conocimiento del Medio Social y Cultural: Balance y propuesta para el Grado en Maestro/a de Educación Primaria. En J. I. Alonso, C. J. Gómez y T. Izquierdo (Eds.), La formación del profesorado en Educación Infantil y Primaria (pp. 305-312). Murcia: Universidad de Murcia.

Ortega, D. (2015). La enseñanza de las Ciencias Sociales, las TIC y el tratamiento de la información y competencia digital (TICD) en el Grado de Maestro/a de Educación Primaria de las universidades de Castilla y León. Enseñanza de las Ciencias Sociales. Revista de Investigación, 14, 121-134.

Ortega, D. y Gómez, I. $M^{a}$ (2015). El MOOC como nueva estrategia/herramienta de enseñanza-aprendizaje en la Didáctica de la Geografía. En $M^{a}$ T. Tortosa, J. D. Álvarez y N. Pellín (Coords.), XIII Jornadas de Redes de Investigación en Docencia Universitaria: Nuevas estrategias organizativas y metodológicas en la formación universitaria para responder a la necesidad de adaptación y cambio (pp. 44-54). Alicante: Universidad de Alicante.

Ortega, D., Gómez, I. Ma y Moreno, J. R. (2016). Competencia digital, digitalización de la formación y MOOC para maestros/as de Geografía de Educación Primaria. En R. Sebastiá y E. Tonda (Eds.), La Investigación e Innovación en la enseñanza de la Geografía (pp. 363-373). Alicante: Universidad de Alicante. 
Osset, J. (Coord.) (2013). WebQuest. Madrid: Ministerio de Educación, Cultura y Deporte. Subdirección General de Documentación y Publicaciones. Recuperado de http://www.ite.educacion.es/formacion/materiales/59/cd/index.htm

Palomares, A. y Garrote, D. (2010). Competencias básicas y nuevo modelo de evaluación. En M. Marín (Coord.), Evaluación de competencias en los nuevos grados (pp. 147-151). Ciudad-Real: Universidad de Castilla-La Mancha.

Pérez, T. D. (2004). La WebQuest como recurso educativo de Ciencias Sociales en Educación Primaria: el castillo de Crevillente. En $M^{a}$ I. Vera y D. Pérez i Pérez (Coord.), Formación de la ciudadanía: las TICs y los nuevos problemas. Alicante: AUPDCS.

Poy, R. y Gonzáles-Aguilar, A. (2014). Factores de éxito de los MOOC: algunas consideraciones críticas. Iberian Journal of Information Systems and Technologies (RISTI), E1, 105-118.

Prendes, M $M^{\text {a }}$ P., Catañeda, L. y Gutiérrez, I. (2010). Competencias para el uso de TIC de los futuros maestros. Comunicar, 18(35), 175-182.

Ramos, L. E. (2009). Utilización de las Webquest como recurso educativo en educación física. Quaderns digitals: Revista de Nuevas Tecnologías y Sociedad, 59. Recuperado de

http://www.quadernsdigitals.net/index.php?accionMenu=hemeroteca.VisualizaNum eroRevistalU.visualiza\&numeroRevista_id=806

Raposo, M. (2014). Orientaciones pedagógicas para los MOOC. En Grupo de Investigación GTEA (Org.), III Workshop internacional sobre Creación de MOOC con anotaciones multimedia. Recuperado de http://gtea.uma.es/congresos/?page_id $=417$

Revuelta, F. y Esnaola, G. (2013). Videojuegos en redes sociales. Perspectivas del edutainment y la pedagogía lúdica en el aula. Barcelona: Laerte.

Rivero, P. (2011). Un estudio sobre la efectividad de la multimedia expositiva para el aprendizaje de la Historia. Enseñanza de las Ciencias Sociales. Revista de investigación, $10,45-50$.

Roblizo, M. y Cózar, R. (2015). Usos y competencias en TIC en los futuros maestros de Educación Infantil y Primaria: hacia una alfabetización tecnológica real para docentes. Pixel Bit. Revista de Medios y Educación, 47, 23-39.

Rowntree, D. (1981). Statistics without tears. London: Penguin.

Sangrà, A. (2011). Estratègies, accions i fases dels processos d'integració de les TIC en la innovació docent universitària. Aloma. Revista de Psicologia, Ciències de l'Educació i de l'Esport, 29, 291-306.

Sevillano, $M^{a}$ L. (coord.). (2007). Investigar para innovar en enseñanza. Madrid: Pearson Prentice Hall.

Temprano, A. (2009). Webquest. Aproximación práctica al uso de Internet en el aula. Sevilla: MAD.

Trigueros, F. J., Molina, S., Sánchez, R., Valverde, I. y Agulló, S. (2010). La competencia digital y la Didáctica de las Ciencias Sociales en la formación permanente del profesorado de Infantil y Primaria. En R. $M^{a}$ Ávila, Ma P. Rivero y P. L. Domínguez (Coords.), Metodología de investigación en didáctica de las ciencias sociales (pp. 553564). Zaragoza: AUPDCS-Institución Fernando el Católico. 
Trigueros, F. J., Sánchez, R. y Vera, Ma I. (2012). El profesorado de Educación Primaria ante las TIC: realidad y retos. REIFOP, 15(1), 101-112.

Vázquez, E., López, E. y Sarasola, J. L. (2013). La expansión del conocimiento en abierto: los MOOC. Barcelona: Octaedro-ICE UB.

Vivancos, J. (2008). Tratamiento de la información y competencia digital. Madrid: Alianza.

Zubillaga del Río, A. y Alba, C. (2005). Las Webquest como recurso educativo para atender la diversidad. Comunicación y pedagogía: Nuevas tecnologías y recursos didácticos, 206, 50-57. 